\title{
Thromboelastography findings in critically ill COVID-19 patients
}

\author{
Nouran Salem ${ }^{1} \cdot$ Bassam Atallah $^{1,2} \cdot$ Wasim S. El Nekidy ${ }^{1,2} \cdot$ Ziad G. Sadik $^{1} \cdot$ Woosup Michael Park $^{3} \cdot$ Jihad Mallat $^{2,4,5}$
}

Accepted: 26 September 2020 / Published online: 4 October 2020

(c) Springer Science+Business Media, LLC, part of Springer Nature 2020

\begin{abstract}
The rate of venous and arterial thrombotic events among patients infected with severe acute respiratory syndrome coronavirus-2 (SAR-CoV-2) is high. This may be due to a hypercoagulable state induced by the severe inflammation that results from the SAR-CoV-2 infection. We aimed to determine hypercoagulable states' incidence based on thromboelastography study and its association with thrombotic events in critically ill patients with coronavirus disease 2019 (COVID-19). Fifty-two COVID-19 patients who had thromboelastography study were retrospectively included. All patients received pharmacologic thromboprophylaxis. The hypercoagulable state was observed in 16 patients (30.8\%). Among them, maximum amplitude and a-angle were elevated in $75 \%$ and $25 \%$, respectively. Reaction time and $\mathrm{K}$ were low in only $12.5 \%$ for both of them. Inflammatory and coagulation markers, as well as thromboprophylaxis regimens, were not associated with a hypercoagulable state. Fourteen patients (27\%) experienced a total of 16 thrombotic events, including 8 (57\%) deep venous thrombosis, 6 (43\%) pulmonary embolism, and $2(14.3 \%)$ arterial thrombosis. The hypercoagulable state was not significantly associated with thrombotic events. In summary, we observed a lower rate of hypercoagulable state on thromboelastography study in critically ill COVID-19 patients. Also, the hypercoagulable state was not associated with the occurrence of thrombotic events.
\end{abstract}

Keywords COVID-19 · Thromboembolism · Thrombotic events · Thromboelastography (TEG) · Hypercoagulable state · Critically ill patients

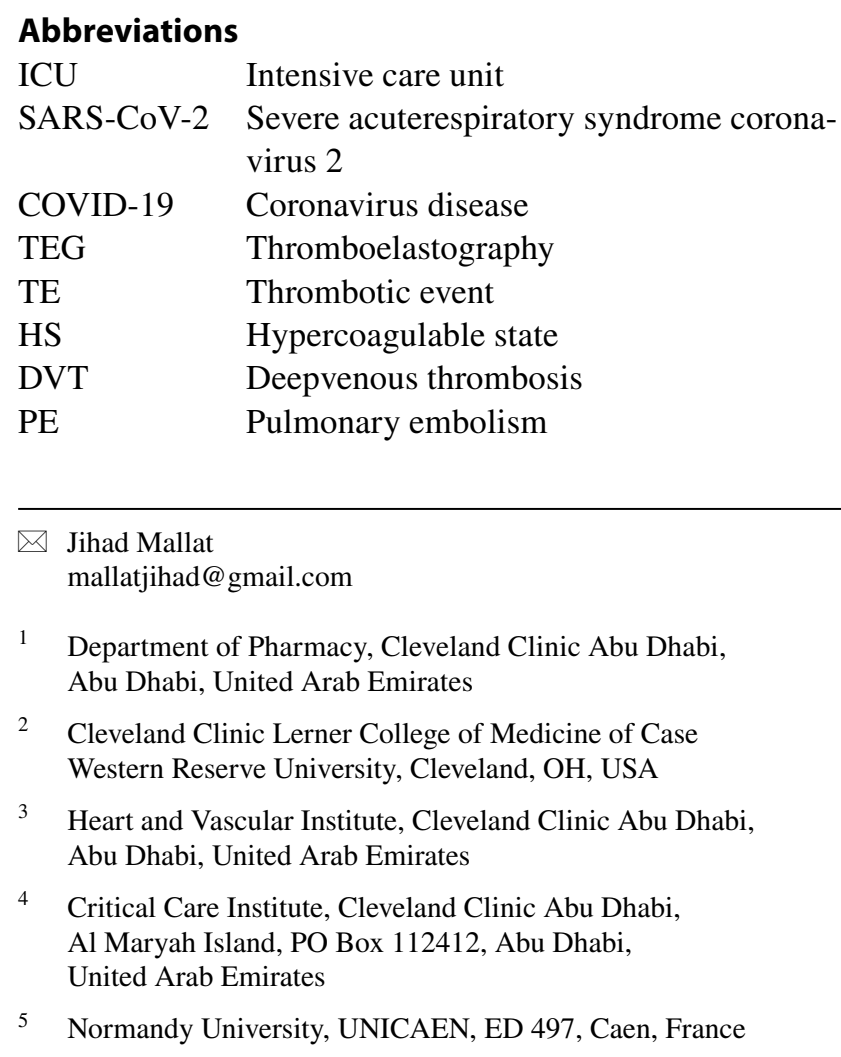

$\begin{array}{ll}\text { VTE } & \text { Venous thromboembolism } \\ \text { LLN } & \text { Lower limit of normal } \\ \text { ULN } & \text { Upper limit of normal } \\ \text { IQR } & \text { Interquartile range } \\ \text { MA } & \text { Maximum amplitude } \\ \text { LY30 } & \text { Lysis at } 30 \text { minutes }\end{array}$

\section{Introduction}

Severe acute respiratory syndrome coronavirus-2 (SARCoV-2) responsible for the coronavirus disease 2019 (COVID-19) has hit the world as a global pandemic at an unprecedented scale. High rates of venous and arterial thrombotic events (TE) were observed in COVID-19 critically ill patients $[1,2]$. The high TE rate may be the result of a hypercoagulable state (HS) induced by the inflammatory reaction induced by SAR-CoV-2. Thromboelastography (TEG) is a point-of-care test explicitly designed to assess overall clotting formation and dissolution in whole blood. A few recent studies showed different rates of HS in COVID19 patients based on the TEG study [1-3]. However, there were conflicting results regarding the association between 
a HS and TE $[1,2]$. The present study's aims were to: (1) explore the incidence of HS using TEG study in COVID-19 patients admitted to the intensive care unit (ICU); (2) assess if HS was associated with TE.

\section{Methods}

The institutional Ethics Committee of Cleveland Clinic Abu Dhabi approved the study (number: A-2020-055), and a waiver of informed consent was obtained. All adult patients admitted to our ICU between April 3 and May 29, 2020, with confirmed SARS-CoV-2 infection (virus detected by a real-time reverse-transcriptase-polymerase-chain-reaction assay of a nasopharyngeal sample) who underwent a TEG study were included. Deidentified data including demographics, laboratory data at or within 48 hours of the TEG study, occurrence of TE during ICU admission, and TEG parameters (reaction times [R], K, $\alpha$-angle, maximum amplitude [MA], and lysis at 30 minutes [LY30]) were collected. All TEG studies were performed with heparinase using the Thromboelastography 6 s Hemostasis Analyzer (Haemonetics, Braintree, MA). HS was defined as MA $>69 \mathrm{~mm}$ (upper limit of normal [ULN]), $\alpha$-angle $>77^{\circ}$ (ULN), $R<4.3 \mathrm{~min}$ (lower limit of normal [LLN]), or $\mathrm{K}<0.8 \mathrm{~min}$ (LLN). TE was defined as venous thromboembolism (VTE) or arterial thrombosis. VTE included deep venous thrombosis (DVT) and pulmonary embolism (PE).

\section{Statistical analysis}

Continuous variables are expressed as median [interquartile range (IQR)], and proportions were used for categorical variables. Mann-Whitney $U$, Chi square, and Fisher exact tests were used for data analyses. P-value $<0.05$ was considered as statistically significant.

\section{Results}

From April 3 to May 29, 2020, 181 adult patients with COVID-19 infection were admitted to the ICU. Among them, 52 patients (29\%) had a TEG study and were included in this analysis. The median age was 53 (IQR 39-62) years, and $98 \%$ of patients were male. All patients received pharmacologic thromboprophylaxis (Table 1) according to our institutional protocol. The median time from ICU admission to a TEG study was 9 (IQR 4-17) days. HS was observed in 16 patients (30.8\%). Among them, MA and $\alpha$-angle were above the ULN in $75 \%$ and $25 \%$, respectively. R and $\mathrm{K}$ were below the LLN in only $12.5 \%$ for both of them. Table 1 shows the comparisons between patients with HS and nonHS. Demographic characteristics, including the ethnic origin, and comorbidities were not differently distributed between the two groups. Lymphocyte count was the only variable with a statistically significance difference, which was lower in the HS group compared to the non-HS group. Inflammatory and coagulation markers were not statistically different between the two groups (Table 1). Vasopressor use, but not thromboprophylaxis regimen, was significantly associated with HS. Fourteen of 52 patients (27\%) experienced a total of 16 TE, including 8 (57\%) DVT, 6 (43\%) PE, and $2(14.3 \%)$ arterial thrombosis. The median time from ICU admission to TE was 7 (IQR: 2-14) days. Thirty-four patients $(65.4 \%)$ underwent 40 VTE imaging studies, of which 24 (60\%) deep venous compression ultrasonography, and $16(40 \%)$ computed tomography pulmonary angiogram. HS was not significantly associated with TE (Table 1). MA, $\alpha$-angle, $\mathrm{R}$, and $\mathrm{K}$ were not significantly different between TE and non-TE groups (Table 2). Only LY30 was statistically lower in the TE group. Regarding laboratory data, only D-dimer was significantly associated with TE (Table 2).

\section{Discussion}

The main findings of our study were: (1) HS was observed in $30.8 \%$ of patients despite thromboprophylaxis; (2) HS was not associated with the occurrence of TE.

TEG has been widely used to detect HS in post-operative and septic patients $[4,5]$. The incidence of HS in our COVID-19 ICU patients of $30.8 \%$ is much lower than what had been previously reported in this patient population (ranging from 50 to 90\%) [1-3].The difference between our HS incidence and the other reports might be explained by the fact that TEG was performed within the first 3 days of ICU admission in the other studies, while it was done at a median of 9 days of ICU admission in our study [1-3]. Another explanation could be the dissimilar populations between our study and the others. Indeed, our population is mainly constituted of patients from India/Pakistan and Arabic countries, whereas patients were from USA/Europe in the other studies [1-3].

In line with the previous reports, HS was due mainly to high MA and $\alpha$-angle suggestive of elevated fibrinogen and platelet activities [1-3]. Thus, the role of antiplatelet treatments in COVID-19 patients should be investigated in further studies.

We found that only low lymphocyte count and vasopressor use were associated with HS, but not inflammatory or coagulation markers. Only one study explored the factors related to HS in COVID-19 patients [2]. The authors observed that only D-dimer was significantly associated with HS, but not inflammatory markers [2].

Surprisingly, we did not find a significant association between HS and TE (Table 1). Our findings are in line with 
Table 1 Comparisons of baseline characteristics, laboratory data, treatments, and outcomes between hypercoagulable state (HS) and non-HS groups

\begin{tabular}{|c|c|c|c|c|}
\hline Variables & All patients $(\mathrm{n}=52)$ & HS $(n=16)$ & Non-HS $(n=36)$ & p-value \\
\hline Age, year & $53[39-62]$ & $52[40-63]$ & $53[39-62]$ & 0.74 \\
\hline Male, n (\%) & $51(98)$ & $16(100)$ & $35(97.2)$ & 1.00 \\
\hline Weight (kg) & 76 [63-87] & $78[62-84]$ & $76[63-88]$ & 0.81 \\
\hline BMI $\left(\mathrm{kg} / \mathrm{m}^{2}\right)$ & $25.8[23.0-29.5]$ & $26.2[23.2-28.0]$ & $25.8[22.7-29.7]$ & 0.91 \\
\hline Race or ethnic origin, $\mathrm{n}(\%)$ & & & & 0.35 \\
\hline Asia (Indian/Pakistan) & $24(46)$ & $5(31)$ & $19(53)$ & \\
\hline Arab & $13(25)$ & $5(31)$ & $8(22)$ & \\
\hline Other & $15(29)$ & $6(37.5)$ & $9(25)$ & \\
\hline Patients with comorbidities, n (\%) & $30(57.7)$ & $8(50)$ & $22(61)$ & 0.45 \\
\hline \multicolumn{5}{|l|}{ Comorbidities distribution, n (\%) } \\
\hline Diabetes mellitus & $20(38.5)$ & $5(31.3)$ & 15 (41.7) & 0.55 \\
\hline Hypertension & $15(28.8)$ & $5(33.3)$ & $10(27)$ & 0.74 \\
\hline Chronic artery disease & $4(7.7)$ & $1(6.3)$ & $3(8.3)$ & 1.00 \\
\hline Chronic kidney disease & $4(7.7)$ & $2(12.5)$ & $2(5.6)$ & 0.58 \\
\hline \multicolumn{5}{|l|}{ Reasons for ICU admission, $\mathrm{n}(\%)$} \\
\hline Acute respiratory distress syndrome & $47(90.4)$ & $13(81.2)$ & $34(94.4)$ & 0.33 \\
\hline \multicolumn{5}{|l|}{ Laboratory data within 48 hours of TEG tests } \\
\hline C-reactive protein, $\mathrm{mg} / \mathrm{L}$ & $49.6[9.0-117.2]$ & $61.4[8.5-210.5]$ & $49.0[9.1-113.8]$ & 0.56 \\
\hline Leucocytes count, $/ \mathrm{mm}^{3}$ & $12,570[8475-18,010]$ & $13,030[9030-21,177]$ & $11,790[8257-14,860]$ & 0.23 \\
\hline Lymphocytes count $\left(\mathrm{mm}^{3}\right)$ & $1080[787-1410]$ & $885[445-1100]$ & $1185[870-1652]$ & 0.015 \\
\hline Neutrophil/lymphocyte ratio & $8.6[4.6-14.4]$ & $13.8[5.1-26.2]$ & $7.7[4.6-11.5]$ & 0.10 \\
\hline Platelet count, $/ \mathrm{mm}^{3}$ & 228 [137-292] & $220[126-420]$ & $230[138-276]$ & 0.44 \\
\hline INR & $1.2[1.1-1.3]$ & $1.2[1.1-1.2]$ & $1.2[1.1-1.4]$ & 0.48 \\
\hline aptt & $35.2[30.6-47.0]$ & $34.6[33.1-37.9]$ & $38.4[27.9-52.0]$ & 0.59 \\
\hline D-dimer, $\mu \mathrm{g} / \mathrm{mL}$ (normal reference: $<0.05$ ) & $4.0[3.3-4.0]$ & $4.0[3.7-4.0]$ & $4.0[3.1-4.0]$ & 0.19 \\
\hline D-dimer $(\max )>2 \mu \mathrm{g} / \mathrm{mL}, \mathrm{n}(\%)$ & $49 / 51(96 \%)$ & $16(100)$ & $33 / 35(94.3)$ & 1.00 \\
\hline Fibrinogen, $\mathrm{g} / \mathrm{L}$ & $4.0[2.7-6.0]$ & $4.1[3.7-6.1]$ & $4.0[2.5-5.2]$ & 0.79 \\
\hline Ferritin, $\mu \mathrm{g} / \mathrm{L}$ (reference range: $36-480$ ) & 806 [666-1317] & $924[674-2028]$ & $784[657-1267]$ & 0.37 \\
\hline Interleukin $6, \mathrm{ng} / \mathrm{L}$ & $657[217-1401]$ & 728 [607-2099] & $607[117-1401]$ & 0.19 \\
\hline \multicolumn{5}{|l|}{ TEG parameters (heparinase) } \\
\hline $\mathrm{R}, \min$ (normal reference: $4.3-8.3$ ) & $8.1[6.7-10.6]$ & $7.4[5.5-12.5]$ & $8.6[7.2-10.6]$ & 0.35 \\
\hline $\mathrm{K}, \min$ (normal reference: $0.8-1.9$ ) & $1.3[1.2-1.9]$ & $1.3[1.0-1.9]$ & $1.3[1.2-1.9]$ & 0.23 \\
\hline$\alpha$-Angle value, degree (normal reference: $64-77$ ) & $72.1[67.2-74.4]$ & $74.3[70.0-77.5]$ & $71.6[66.4-73.6]$ & 0.014 \\
\hline Maximum amplitude, mm (normal reference: 52-69) & $65.8[59.6-68.7]$ & $70.0[68.6-71.7]$ & $63.2[56.8-66.7]$ & $<0.001$ \\
\hline LY30, \% (normal reference: $0.0-2.2$ ) & $0.0[0.0-0.1]$ & $0.0[0.0-0.7]$ & $0.0[0.0-0.2]$ & 0.66 \\
\hline \multicolumn{5}{|l|}{ ICU treatments, $\mathrm{n}(\%)$} \\
\hline Invasive mechanical ventilation & $46(88.5)$ & $14(87.5)$ & $32(88.9)$ & 1.00 \\
\hline Prone position & $34(65.4)$ & $11(68.8)$ & $23(63.9)$ & 1.00 \\
\hline Muscular blocker agents & $42(80.8)$ & $14(87.5)$ & $28(77.8)$ & 0.70 \\
\hline Vasopressor support & $42(80.8)$ & $16(100)$ & $26(72.2)$ & 0.022 \\
\hline Renal replacement therapy & $16(30.8)$ & $6(37.5)$ & $10(27.8)$ & 0.53 \\
\hline ECMO & $7(13.5)$ & $1(6.3)$ & $6(16.7)$ & 0.41 \\
\hline Tocilizumab & $44(84.6)$ & $14(87.5)$ & $30(83.8)$ & 1.00 \\
\hline Thrombotic events, n (\%) & $14(26.9)$ & $3(18.8)$ & $11(30.6)$ & 0.51 \\
\hline Thromboprophylaxis strategy, n (\%) & & & & 0.69 \\
\hline Standard prophylactic dose & $10(19.2)$ & $2(12.5)$ & $98(22.2)$ & \\
\hline High-intensity prophylactic dose & $32(61.5)$ & $11(68.8)$ & $21(58.3)$ & \\
\hline Therapeutic anticoagulation & $10(19.2)$ & $3(18.8)$ & $7(19.4)$ & \\
\hline ICU mortality, n (\%) & $17(32.7)$ & $6(37.5)$ & $11(30.6)$ & 0.75 \\
\hline
\end{tabular}

Bold values indicate that the comparisons are statistically significant $(\mathrm{p}<0.05)$

Data are expressed as median [interquartile range] or count (percentage)

$I C U$ intensive care unit, $R$ reaction time, $L Y 30$ lysis at 30 minutes, TEG thromboelastography, ECMO extracorporeal membrane oxygenation, $H S$ hypercoagulability state 
Table 2 Comparisons of thromboelatography parameters between thrombotic events (TE) and non-TE groups

\begin{tabular}{|c|c|c|c|}
\hline Variables & $\mathrm{TE}(\mathrm{n}=14)$ & Non-TE $(n=38)$ & p-value \\
\hline \multicolumn{4}{|l|}{ Laboratory data within $48 \mathrm{~h}$ of TEG } \\
\hline C-reactive protein, $\mathrm{mg} / \mathrm{L}$ & $50.2[5.1-177.2]$ & $49.0[9.7-115.1]$ & 0.87 \\
\hline Leucocytes count, $/ \mathrm{mm}^{3}$ & $13,470[9705-15,152]$ & $12,260[8302-18,432]$ & 0.73 \\
\hline Lymphocytes count $\left(\mathrm{mm}^{3}\right)$ & $1265[877-1587]$ & $1020[667-1275]$ & 0.24 \\
\hline Lymphocytes $\leq 1000 / \mathrm{mm}^{3}, \mathrm{n}(\%)$ & $5(35.7)$ & $17(44.7)$ & 0.75 \\
\hline Neutrophil/lymphocyte ratio & $7.2[5.3-10.6]$ & $9.1[4.4-15.8]$ & 0.73 \\
\hline Platelet count, $/ \mathrm{mm}^{3}$ & $266[175-373]$ & 210 [125-269] & 0.11 \\
\hline INR & $1.2[1.0-1.4]$ & $1.2[1.1-1.3]$ & 1.00 \\
\hline aptt & $49.4[29.0-66.0]$ & $35.1[31.2-42.1]$ & 0.13 \\
\hline D-dimer, $\mu \mathrm{g} / \mathrm{mL}$ (normal reference: $<0.05$ ) & $4.0[4.0-4.0]$ & $1.8[0.8-3.0]$ & $<0.001$ \\
\hline Fibrinogen, $\mathrm{g} / \mathrm{L}$ & $4.0[2.4-6.1]$ & $4.0[2.9-6.0]$ & 0.93 \\
\hline Ferritin, $\mu \mathrm{g} / \mathrm{L}$ (reference range: $36-480$ ) & $784[600-1482]$ & 860 [665-1295] & 0.77 \\
\hline Interleukin $6, \mathrm{ng} / \mathrm{L}$ & $1097[322-1645]$ & 634 [215-1339] & 0.28 \\
\hline \multicolumn{4}{|l|}{ TEG parameters } \\
\hline $\mathrm{R}$, min (normal reference: $4.3-8.3$ ) & $7.7[7.3-10.8]$ & $8.5[6.2-10.8]$ & 0.59 \\
\hline $\mathrm{R}<4.3 \min , \mathrm{n}(\%)$ & $0(0)$ & $2(5.3)$ & 1.00 \\
\hline $\mathrm{K}$, min (normal reference: $0.8-1.9$ ) & $1.3[1.2-1.9]$ & $1.5[1.2-1.9]$ & 0.78 \\
\hline $\mathrm{K}<0.8 \min , \mathrm{n}(\%)$ & $0(0)$ & $2(5.3)$ & 1.00 \\
\hline$\alpha$ Angle value, degree (normal reference: $64-77$ ) & $73.2[68.0-74.8]$ & $70.9[67.0-74.3]$ & 0.66 \\
\hline$\alpha$-Angle $>77$ degree, $\mathrm{n}(\%)$ & $0(0)$ & $4(10.5)$ & 0.56 \\
\hline Maximum amplitude, mm (normal reference: 52-69) & $66.7[61.4-68.1]$ & $65.2[59.4-68.9]$ & 0.67 \\
\hline Maximum amplitude $>69$ mm, $\mathrm{n}(\%)$ & $3(21.4)$ & $9(23.7)$ & 1.00 \\
\hline LY30, \% (normal reference: 0.0-2.2) & $0.0[0.0-0.0]$ & $0.0[0.0-0.2]$ & 0.041 \\
\hline Thromboprophylaxis strategy, n (\%) & & & 0.06 \\
\hline Standard prophylactic dose & $5(35.7)$ & $5(13.2)$ & \\
\hline High-intensity prophylactic dose & $5(35.7)$ & $27(71.1)$ & \\
\hline Therapeutic anticoagulation & $4(28.6)$ & $6(15.8)$ & \\
\hline
\end{tabular}

Bold values indicate that the comparisons are statistically significant $(\mathrm{p}<0.05)$

$R$ reaction time, $L Y 30$ lysis at 30 minutes, TEG thromboelastography, TE thrombotic events. Data are expressed as median [interquartile range] or count (percentage)

those of a recent study, which observed a rate of $34 \%$ of VTE in patients with $\mathrm{HS}$ as compared to $28 \%$ in patients with no HS $(p=0.59)$ [2]. However, Mortus et al. observed a HS in $100 \%$ of patients with $\geq 2$ TE (high TE) [1]. Nevertheless, the sample size of that study was very small (21 patients). Also, central venous and dialysis lines thrombosis were included in the TE, as opposed to our study. Furthermore, the MA after heparinase correction was not statistically different between the low $(\leq 1)$ and high TE groups $(72 \pm 11$ vs. $77 \pm 7 \mathrm{~mm}$, respectively, $\mathrm{p}=0.23)$ [1].

Only D-dimer and LY30 were significantly associated with TE (Table 2). The low LY30 value represents a shutdown of the fibrinolysis process. Wright et al. found that LY30 significantly predicted VTE occurrence, with an area under the ROC curve of $0.742(p=0.022)$, in COVID19 patients [6]. Increased angiotensin II expression and consequently increased plasminogen activator inhibitor-1 expression might be responsible for the reduced fibrinolysis in COVID-19 patients.

The single-center, retrospective design and small sample size is a limitation to this study. Additionally, TEG study was performed only once in some patients, which limits evaluation of coagulable status during the whole ICU stay. Also, not all patients underwent TE imaging, which might have resulted in missing a TE diagnosis in some patients.

\section{Conclusions}

In summary, we observed a lower rate of HS on TEG in ICU COVID-19 patients. HS was not associated with the occurrence of TE. 
Author contributions JM, NS, and WMP designed the study. BA, NS, ZGS and WSE collected data. JM conducted statistical analyses. $\mathrm{JM}$, NS, and BA participated in manuscript writing and reviewing. Critical revision of the manuscript for important intellectual content: WMP, NS, BA, ZGS, and WSE. All authors read and approved the final manuscript.

\section{Funding None.}

Data availability The datasets generated and/or analyzed during the current study are not publicly available due to privacy (patients' data) but are available from the corresponding author on reasonable request.

\section{Compliance with ethical standards}

Conflict of interest The authors declare that they have no competing interest.

Ethical approval The study was approved by the Clinical Research Ethics Committee of Cleveland Clinic Abu Dhabi (Number: A-2020-055) and consent was waived due to the observational nature of the study.

\section{References}

1. Mortus JR, Manek SE, Brubaker LS, Loor M, Cruz MA, Trautner BW, Rosengart TK (2020) Thromboelastographic results and hypercoagulability syndrome in patients with coronavirus disease
2019 who are critically IlI. JAMA Netw Open 3:e2011192. https ://doi.org/10.1001/jamanetworkopen.2020.11192

2. Yuriditsky E, Horowitz JM, Merchan C, Ahuja T, Brosnahan SB, McVoy L, Berger JS (2020) Thromboelastography profiles of critically Ill patients with coronavirus disease 2019. Crit Care Med 48(9):1319-1326. https://doi.org/10.1097/CCM.0000000000 004471

3. Panigada M, Bottino N, Tagliabue P, Grasselli G, Novembrino C, Chantarangkul V, Pesenti A, Peyvandi F, Tripodi A (2020) Hypercoagulability of COVID-19 patients in intensive care unit: a report of thromboelastography findings and other parameters of hemostasis. J Thromb Haemost 18(7):1738-1742. https://doi. org/10.1111/jth. 14850

4. Hincker A, Feit J, Sladen RN, Wagener G (2014) Rotational thromboelastometry predicts thromboembolic complications after major non-cardiac surgery. Crit Care 18(5):549. https://doi. org/10.1186/s13054-014-0549-2

5. Müller MC, Meijers JC, Vroom MB, Juffermans NP (2014) Utility of thromboelastography and/or thromboelastometry in adults with sepsis: a systematic review. Crit Care 18(1):R30. https://doi. org/10.1186/cc13721

6. Wright FL, Vogler TO, Moore EE, Moore HB, Wohlauer MV, Urban S, Nydam TL, Moore PK, McIntyre RC Jr (2020) Fibrinolysis shutdown correlation with thromboembolic events in severe COVID-19 infection. J Am Coll Surg 231(2):193-203.e1. https:// doi.org/10.1016/j.jamcollsurg.2020.05.007

Publisher's Note Springer Nature remains neutral with regard to jurisdictional claims in published maps and institutional affiliations. 